PROCEEDINGS OF THE

AMERICAN MATHEMATICAL SOCIETY

Volume 129, Number 1, Pages 155-163

S 0002-9939(00)05495-2

Article electronically published on June 21, 2000

\title{
SEPARATED SEQUENCES IN NONREFLEXIVE BANACH SPACES
}

\author{
ANDRZEJ KRYCZKA AND STANISEAW PRUS
}

(Communicated by Dale Alspach)

\begin{abstract}
We prove that there is $c>1$ such that the unit ball of any nonreflexive Banach space contains a $c$-separated sequence. The supremum of these constants $c$ is estimated from below by $\sqrt[5]{4}$ and from above approximately by 1.71. Given any $p>1$, we also construct a nonreflexive space so that if the convex hull of a sequence is sufficiently close to the unit sphere, then its separation constant does not exceed $2^{1 / p}$.
\end{abstract}

\section{INTRODUCTION}

Sequences of separated elements appear quite often in the literature on Banach spaces. They are strongly related to the problem of packing balls (see [15]), average distances (see [2]) and infinite dimensional convexity (see [9]). The last notion in turn plays an important role in metric fixed point theory (see [8] and [1]).

A sequence is said to be $r$-separated if distances between its elements are bounded from below by $r$. One of the basic general results on separated sequences is due to Elton and Odell 7 . They proved that the unit ball of an arbitrary infinite dimensional Banach space contains an $r$-separated sequence for some $r>1$. In this paper we show that there exists a constant $c>1$ such that in the unit ball of any nonreflexive Banach space one can find a $c$-separated sequence. On the other hand for each $1<p<\infty$ we construct a nonreflexive Banach space $Y_{p}$ whose unit ball does not contain a sequence with the separation constant greater than $2^{1 / p}$ such that its convex hull is arbitrarily close to the unit sphere. These results provide answers to two of the questions posed in 22. Let us however mention that our notation does not coincide with that of [2].

\section{Preliminaries}

Let $X$ be a Banach space. By the separation constant of a sequence $\left(x_{n}\right)$ in $X$ we mean the number

$$
\operatorname{sep}\left(x_{n}\right)=\inf \left\{\left\|x_{m}-x_{n}\right\|: m \neq n\right\} .
$$

Received by the editors October 29, 1998 and, in revised form, March 14, 1999.

1991 Mathematics Subject Classification. Primary 46B20.

Key words and phrases. Nonreflexive spaces, separation measure of noncompactness, James' space. 
Having a bounded set $A \subset X$, we put

$$
\beta(A)=\sup \left\{\operatorname{sep}\left(x_{n}\right):\left\{x_{n}\right\} \subset A\right\} .
$$

This quantity is called the separation measure of noncompactness of $A$ (see [1]).

By $B_{X}$ we denote the closed unit ball of the space $X$. Kottman 14 introduced the packing constant $P\left(\aleph_{0}, X\right)$ as the supremum of all numbers $r>0$ such that there exists a sequence of pairwise disjoint balls of radius $r$ contained in $B_{X}$. We have

$$
P\left(\aleph_{0}, X\right)=\frac{\beta\left(B_{X}\right)}{2+\beta\left(B_{X}\right)}
$$

(see [14]).

Let $A$ be a subset of $X$. The closed convex hull of $A$ will be denoted by co $A$. Assuming that the space $X$ is infinite dimensional and $0 \leq \epsilon<\beta\left(B_{X}\right)$, we set

$$
\Delta(\epsilon)=\inf \left\{1-\inf \left\{\|x\|: x \in \operatorname{co}\left\{x_{n}\right\}\right\}\right\}
$$

where the first infimum is taken over all sequences $\left(x_{n}\right)$ in $B_{X}$ with $\operatorname{sep}\left(x_{n}\right) \geq$ $\epsilon$. The function $\Delta$ is called the modulus of noncompact convexity of $X$. It is nonnegative and $\Delta(0)=0 . \Delta$ can be treated as an infinite dimensional counterpart of the classical modulus of convexity (see [5]). We will also consider the following coefficient:

$$
S_{0}(X)=\sup \left\{\epsilon \in\left[0, \beta\left(B_{X}\right)\right): \Delta(\epsilon)=0\right\} .
$$

We have the formula $\beta\left(B_{l_{p}}\right)=2^{1 / p}$ for $1 \leq p<\infty$ (see [4] or [15], p. 91). This coefficient may be therefore arbitrarily close to 1 . On the other hand $\beta\left(B_{c_{0}}\right)=2$ and moreover $S_{0}\left(l_{1}\right)=S_{0}\left(c_{0}\right)=2$. It follows that $\beta\left(B_{X}\right)=S_{0}(X)=2$ for any Banach space $X$ containing an isomorphic copy of $l_{1}$ or $c_{0}$ (see [13]).

\section{REsults}

Theorem 1. There exists a constant $c>1$ such that if $X$ is a nonreflexive Banach space, then $\beta\left(B_{X}\right) \geq c$.

Proof. Let $X$ be a nonreflexive Banach space. By a result of James 12 for every $\theta \in(0,1)$ there are two sequences: $\left(x_{n}\right)$ in $B_{X}$ and $\left(x_{n}^{*}\right)$ in $B_{X^{*}}$ such that $x_{k}^{*}\left(x_{i}\right)=\theta$ if $k \leq i$ and $x_{k}^{*}\left(x_{i}\right)=0$ if $i<k$.

Let us put $\epsilon_{1}^{1}=1, \epsilon_{1}^{2}=-1, \epsilon_{i}^{j}=(-1)^{i}$ if $1 \leq j<i$ or $j=2 i$ and $\epsilon_{i}^{j}=(-1)^{i+1}$ if $i \leq j<2 i$ where $i=2,3,4$. Passing to a subsequence, we can assume that there exist positive constants $a_{1}, a_{2}, a_{3}, a_{4}$ for which

$$
\theta a_{i} \leq\left\|\sum_{j=1}^{2 i} \epsilon_{i}^{j} x_{n_{j}}\right\| \leq a_{i}
$$


for any $n_{1}<n_{2}<\cdots<n_{2 i}$ where $i=1,2,3,4$ (see [3]). We consider the following elements:

$$
\begin{aligned}
& x_{n}^{0}=x_{n}, \\
& x_{n}^{1}=\frac{1}{a_{1}}\left(x_{2 n-1}-x_{2 n}\right), \\
& x_{n}^{2}=\frac{1}{a_{2}}\left(x_{1}-x_{3 n-1}-x_{3 n}+x_{3 n+1}\right), \\
& x_{n}^{3}=\frac{1}{a_{3}}\left(-x_{1}-x_{2}+x_{4 n-1}+x_{4 n}+x_{4 n+1}-x_{4 n+2}\right), \\
& x_{n}^{4}=\frac{1}{a_{4}}\left(x_{1}+x_{2}+x_{3}-x_{5 n-1}-x_{5 n}-x_{5 n+1}-x_{5 n+2}+x_{5 n+3}\right)
\end{aligned}
$$

for $n \in \mathbb{N}$. They are contained in $B_{X}$. Moreover, $\operatorname{sep}\left(x_{n}^{0}\right) \geq \theta a_{1}$ and $\operatorname{sep}\left(x_{n}^{i}\right) \geq \frac{\theta a_{i+1}}{a_{i}}$ if $i=1,2,3$. We also have the estimate

$$
\left\|x_{m}^{4}-x_{n}^{4}\right\| \geq x_{5 m+3}^{*}\left(x_{m}^{4}-x_{n}^{4}\right)=\frac{4 \theta}{a_{4}}
$$

whenever $m<n$. It follows that

$$
\beta\left(B_{X}\right) \geq \max \left\{\theta a_{1}, \frac{\theta a_{2}}{a_{1}}, \frac{\theta a_{3}}{a_{2}}, \frac{\theta a_{4}}{a_{3}}, \frac{4 \theta}{a_{4}}\right\} \geq \theta \sqrt[5]{4} .
$$

Since $\theta \in(0,1)$ is arbitrary, we obtain the inequality $\beta\left(B_{X}\right) \geq \sqrt[5]{4}$.

Corollary 1. There exists a constant $C>\frac{1}{3}$ such that if $X$ is a nonreflexive Banach space, then $P\left(\aleph_{0}, X\right) \geq C$.

We do not know what is the greatest possible value of the constant $c$ in Theorem 11 From a remark made by Kottman [14] it follows that if $X$ is James' space (see [11]), then $\beta\left(B_{X}\right)<2$. We will calculate the exact value of this coefficient for a generalized James' space.

Let us first establish some notation. By an interval of natural numbers we mean a set of the form $[m, n]=\{k \in \mathbb{N}: m \leq k \leq n\}$ where $m \leq n$ are natural numbers. Having such intervals $I_{1}, I_{2}$, we write $I_{1} \leq I_{2}$ if $\max I_{1} \leq \min I_{2}$. Let now $I=[m, n]$ and $x=(x(k))$ be a sequence of real numbers. We put

$$
\begin{aligned}
& \delta(I, x)=x(n)-x(m), \quad \delta_{+}(I, x)=\frac{1}{2}(|\delta(I, x)|+\delta(I, x)), \\
& \delta_{-}(I, x)=\frac{1}{2}(|\delta(I, x)|-\delta(I, x)) .
\end{aligned}
$$

We fix $1<p<\infty$. Following James' construction [11, we introduce the space $J_{p}$ of all real sequences $x=(x(k))$ converging to 0 and such that

$$
\|x\|=\sup \left\{\left(\sum_{k=1}^{n}\left|\delta\left(I_{k}, x\right)\right|^{p}\right)^{1 / p}: I_{1} \leq I_{2} \leq \cdots \leq I_{n}\right\}
$$

is finite. This formula defines a norm on $J_{p}$. It is easy to see that the vectors $e_{n}=(0, \ldots, 0,1,0, \ldots)$, where 1 occupies $n$th place, form a basis of $J_{p}$. The basis $\left(e_{n}\right)$ is not boundedly complete (see [10]) and consequently $J_{p}$ is not reflexive. Having an arbitrary sequence $x=(x(k))$ of real numbers and $n \in \mathbb{N}$, we set

$$
P_{n} x=(x(1), \ldots, x(n), 0,0, \ldots)
$$


and $R_{n} x=x-P_{n} x$. $\quad P_{n}$ restricted to $J_{p}$ is a norm-one projection. Moreover, $\lim _{n \rightarrow \infty}\left\|R_{n} x\right\|=0$ for every $x \in J_{p}$. In the sequel we will identify a number $\alpha \in \mathbb{R}$ with a constant sequence $(\alpha, \alpha, \ldots)$.

Lemma 1. Let $\left(x_{n}\right)$ be a bounded sequence in $J_{p}$. For every $\gamma>0$ there exist a subsequence $\left(x_{n_{k}}\right)$, an increasing sequence $\left(m_{k}\right)$ of natural numbers and a constant $\alpha \in \mathbb{R}$ such that

$$
\left\|x_{n_{k}}-\left(P_{m_{1}} x_{n_{1}}+P_{m_{2 k}} R_{m_{1}} \alpha+P_{m_{2 k+1}} R_{m_{2 k}} x_{n_{k}}\right)\right\| \leq \gamma
$$

for every $k \in \mathbb{N}$.

Proof. Since the sequence $\left(x_{n}\right)$ is bounded, passing to a subsequence, we can assume that for each $i \in \mathbb{N}$ there exists $x(i)=\lim _{n \rightarrow \infty} x_{n}(i)$. It is easy to see that $(x(n))$ is a Cauchy sequence, so it has a limit $\alpha$. Clearly $x-\alpha \in J_{p}$.

Therefore, having $\gamma>0$, we can find $m_{1}$ such that $\left\|R_{m_{1}}(x-\alpha)\right\| \leq \frac{1}{6} \gamma$. Next, we put $m_{2}=m_{1}+1$ and choose $n_{1}$ so that $\left\|P_{m_{2}}\left(x_{n_{1}}-x\right)\right\| \leq \frac{1}{6} \gamma$. There is $m_{3}>m_{2}$ for which $\left\|R_{m_{3}} x_{n_{1}}\right\| \leq \frac{1}{6} \gamma$.

Proceeding in this way, we obtain sequences $\left(m_{k}\right),\left(n_{k}\right)$ such that

$$
\left\|P_{m_{2 k}}\left(x_{n_{k}}-x\right)\right\| \leq \frac{1}{6} \gamma \quad \text { and } \quad\left\|R_{m_{2 k+1}} x_{n_{k}}\right\| \leq \frac{1}{6} \gamma
$$

for every $k \in \mathbb{N}$. Then

$$
\begin{aligned}
\| x_{n_{k}}-P_{m_{1}} x_{n_{1}}- & P_{m_{2 k}} R_{m_{1}} \alpha-P_{m_{2 k+1}} R_{m_{2 k}} x_{n_{k}} \| \\
& \leq\left\|P_{m_{1}}\left(x_{n_{k}}-x_{n_{1}}\right)\right\|+\left\|P_{m_{2 k}} R_{m_{1}}\left(x_{n_{k}}-\alpha\right)\right\|+\left\|R_{m_{2 k+1}} x_{n_{k}}\right\| \\
& \leq\left\|P_{m_{1}}\left(x_{n_{1}}-x\right)\right\|+\left\|P_{m_{2 k}} R_{m_{1}}(x-\alpha)\right\|+\frac{4}{6} \gamma \leq \gamma .
\end{aligned}
$$

Theorem 2. Let $1<p<\infty$. Then $\beta\left(B_{J_{p}}\right)=\left(1+2^{p-1}\right)^{1 / p}$.

Proof. Clearly $\left\|2^{-1 / p} e_{n}\right\|=1$ and

$$
\left\|2^{-1 / p} e_{m}-2^{-1 / p} e_{n}\right\|=\left(1+2^{p-1}\right)^{1 / p}
$$

for any $m \neq n$. Consequently $\beta\left(B_{J_{p}}\right) \geq\left(1+2^{p-1}\right)^{1 / p}$.

In order to prove the opposite inequality, we take a sequence $\left(x_{n}\right)$ in $B_{J_{p}}$ and $\gamma \in(0,1)$. By Lemma 1 we obtain corresponding sequences $\left(n_{k}\right),\left(m_{k}\right)$ and a constant $\alpha$. Let us put

$$
\begin{aligned}
& u_{1}=P_{m_{1}} x_{n_{1}}+P_{m_{2}} R_{m_{1}} \alpha+P_{m_{3}} R_{m_{2}} x_{n_{1}}, \\
& u_{2}=P_{m_{1}} x_{n_{1}}+P_{m_{4}} R_{m_{1}} \alpha+P_{m_{5}} R_{m_{4}} x_{n_{2}}
\end{aligned}
$$

and $w=u_{2}-u_{1}$

There is a sequence $I_{1} \leq I_{2} \leq \cdots \leq I_{n}$ of intervals of natural numbers such that

$$
((1-\gamma)\|w\|)^{p} \leq \sum_{k=1}^{n}\left|\delta\left(I_{k}, w\right)\right|^{p} .
$$

Clearly $w(i)=0$ if $i \leq m_{2}$. Therefore we can assume that $m_{2} \leq \min I_{1}$. We can also assume that there is an interval $I_{s}=\left[j_{1}, j_{2}\right]$ for which $j_{1} \leq m_{3}<j_{2}$. 
If $k<s$, then $\delta\left(I_{k}, w\right)=-\delta\left(I_{k}, u_{1}\right)$ and if $k>s$, then $\delta\left(I_{k}, w\right)=\delta\left(I_{k}, u_{2}\right)$. It follows that

$$
\sum_{k<s}\left|\delta\left(I_{k}, w\right)\right|^{p}+\left|u_{1}\left(j_{1}\right)\right|^{p} \leq\left\|u_{1}\right\|^{p} \leq(1+\gamma)^{p}
$$

and

$$
\left|\alpha-u_{2}\left(j_{2}\right)\right|^{p}+\sum_{k>s}\left|\delta\left(I_{k}, w\right)\right|^{p} \leq(1+\gamma)^{p}
$$

Here we treat a sum over the empty set as 0 . Consequently

$$
\begin{aligned}
((1-\gamma)\|w\|)^{p} & \leq 2(1+\gamma)^{p}-\left|u_{1}\left(j_{1}\right)\right|^{p}+\left|u_{2}\left(j_{2}\right)-\left(\alpha-u_{1}\left(j_{1}\right)\right)\right|^{p}-\left|\alpha-u_{2}\left(j_{2}\right)\right|^{p} \\
& \leq 2(1+\gamma)^{p}+\left(1-2^{1-p}\right)\left|u_{1}\left(j_{1}\right)+u_{2}\left(j_{2}\right)-\alpha\right|^{p} .
\end{aligned}
$$

But

$$
2^{1-p}\left|2 u_{1}\left(j_{1}\right)-\alpha\right|^{p} \leq\left|u_{1}\left(j_{1}\right)\right|^{p}+\left|\alpha-u_{1}\left(j_{1}\right)\right|^{p} \leq\left\|u_{1}\right\|^{p} \leq(1+\gamma)^{p}
$$

and similarly

$$
2^{1-p}\left|2 u_{2}\left(j_{2}\right)-\alpha\right|^{p} \leq(1+\gamma)^{p}
$$

Hence

$$
\left|u_{1}\left(j_{1}\right)+u_{2}\left(j_{2}\right)-\alpha\right| \leq 2^{1-1 / p}(1+\gamma)
$$

which gives us the inequality $((1-\gamma)\|w\|)^{p} \leq(1+\gamma)^{p}\left(1+2^{p-1}\right)$. On the other hand $\left\|x_{n_{1}}-x_{n_{2}}\right\| \leq\|w\|+2 \gamma$. Therefore

$$
\operatorname{sep}\left(x_{n}\right) \leq(1-\gamma)^{-1}(1+\gamma)\left(1+2^{p-1}\right)^{1 / p}+2 \gamma .
$$

Since $\gamma>0$ is arbitrary, we obtain $\operatorname{sep}\left(x_{n}\right) \leq\left(1+2^{p-1}\right)^{1 / p}$.

Let us notice that the minimal value of $\left(1+2^{p-1}\right)^{1 / p}$ is approximately equal to 1.71. It is an upper bound for the constant $c$ in Theorem 1 Our proof of Theorem 1 gives $c=\sqrt[5]{4}$ which is about 1.32 .

In [6] it was shown that if $S_{0}(X)<1$, then the space $X$ is reflexive. In contrast to Theorem 1 we will prove that 1 is the greatest lower bound of the values of $S_{0}(X)$ for nonreflexive spaces $X$.

Having $x \in J_{p}$, we put

$$
\begin{aligned}
& |x|_{+}=\sup \left\{\left(\sum_{k=1}^{n}\left(\delta_{+}\left(I_{k}, x\right)\right)^{p}\right)^{1 / p}: I_{1} \leq I_{2} \leq \cdots \leq I_{n}\right\}, \\
& |x|_{-}=\sup \left\{\left(\sum_{k=1}^{n}\left(\delta_{-}\left(I_{k}, x\right)\right)^{p}\right)^{1 / p}: I_{1} \leq I_{2} \leq \cdots \leq I_{n}\right\}
\end{aligned}
$$

and

$$
\|x\|_{ \pm}=\left(|x|_{+}^{p}+|x|_{-}^{p}\right)^{1 / p}
$$

This formula gives an equivalent norm on $J_{p}$. Namely

$$
\|x\| \leq\|x\|_{ \pm} \leq 2^{1 / p}\|x\|
$$

for every $x \in J_{p}$. The space $J_{p}$ with the norm $\|\cdot\|_{ \pm}$will be denoted by $Y_{p}$. Considering the sequence $\left(2^{-1 / p} e_{n}\right)$, we see that $\beta\left(B_{Y_{p}}\right) \geq\left(1+2^{p-1}\right)^{1 / p}$.

Theorem 3. Let $1<p<\infty$. Then $S_{0}\left(Y_{p}\right)=2^{1 / p}$. 
Proof. Let us consider the sequence of vectors $y_{n}=\sum_{k=1}^{n} e_{k}$. Clearly $\left\|y_{n}\right\|_{ \pm}=1$ and $\left\|y_{m}-y_{n}\right\|_{ \pm}=2^{1 / p}$ for any $m \neq n$. It is also easy to see that $\|x\|_{ \pm}=1$ for every $x \in \operatorname{co}\left\{y_{n}\right\}$. This shows that $S_{0}\left(Y_{p}\right) \geq 2^{1 / p}$.

We will show the opposite inequality. To this end we take $\epsilon \in\left(2^{1 / p}, \beta\left(B_{Y_{p}}\right)\right)$ and choose $\gamma>0$ so that

$$
\gamma \leq \frac{1}{3}\left(8^{-p}\left(\epsilon^{p}-2\right)\right)^{p}
$$

Now let $\left(x_{n}\right)$ be a sequence in $B_{Y_{p}}$ with $\operatorname{sep}\left(x_{n}\right) \geq \epsilon$. From Lemma 1 we obtain sequences $\left(n_{k}\right),\left(m_{k}\right)$ and a constant $\alpha$ such that

$$
\left\|x_{n_{k}}-u_{k}\right\|_{ \pm} \leq \frac{\gamma}{p 2^{p}}
$$

for every $k \in \mathbb{N}$, where $u_{k}=P_{m_{1}} x_{n_{1}}+P_{m_{2 k}} R_{m_{1}} \alpha+P_{m_{2 k+1}} R_{m_{2 k}} x_{n_{k}}$. We set $u=P_{m_{1}} x_{n_{1}}+P_{m_{2}} R_{m_{1}} \alpha$ and $z_{N}=\frac{1}{N} \sum_{k=1}^{N} u_{k}$ where $N \in \mathbb{N}$. Clearly it suffices to consider the case when $\alpha \geq 0$.

We will show that

$$
\left\|\frac{1}{N} \sum_{k=1}^{N} x_{n_{k}}\right\|_{ \pm} \leq\|u\|_{ \pm}+2 \gamma
$$

for some $N \in \mathbb{N}$. It is easy to see that

$$
\left\|\frac{1}{N} \sum_{k=1}^{N} P_{m_{2 k+1}} R_{m_{2 k}} x_{n_{k}}\right\|^{p} \leq \frac{2^{p-1}}{N^{p}} \sum_{k=1}^{N}\left\|P_{m_{2 k+1}} R_{m_{2 k}} x_{n_{k}}\right\|^{p} \leq \frac{2^{2 p-1}}{N^{p-1}}
$$

for every $N \in \mathbb{N}$. Therefore we can find $N$ such that

$$
\left\|\frac{1}{N} \sum_{k=1}^{N} P_{m_{2 k+1}} R_{m_{2 k}} x_{n_{k}}\right\|_{ \pm} \leq \gamma .
$$

But one can easily check that

$$
\left\|\frac{1}{N} \sum_{k=1}^{N}\left(P_{m_{1}} x_{n_{1}}+P_{m_{2 k}} R_{m_{1}} \alpha\right)\right\|_{ \pm}=\|u\|_{ \pm} .
$$

Hence

$$
\left\|\frac{1}{N} \sum_{k=1}^{N} x_{n_{k}}\right\|_{ \pm} \leq\left\|z_{N}\right\|_{ \pm}+\gamma \leq\|u\|_{ \pm}+2 \gamma .
$$

Our next aim is to prove that

$$
\|u\|_{ \pm}^{p}<1-\frac{1}{3}\left(8^{-p}\left(\epsilon^{p}-2\right)\right)^{p} .
$$

There are sequences $T_{1}^{1} \leq T_{2}^{1} \leq \cdots \leq T_{r}^{1}$ and $T_{1}^{2} \leq T_{2}^{2} \leq \cdots \leq T_{s}^{2}$ of intervals for which

$$
(1-\gamma)|u|_{+}^{p} \leq \sum_{k=1}^{r}\left(\delta_{+}\left(T_{k}^{1}, u\right)\right)^{p}, \quad(1-\gamma)|u|_{-}^{p} \leq \sum_{k=1}^{s}\left(\delta_{-}\left(T_{k}^{2}, u\right)\right)^{p} .
$$

Since $\alpha \geq 0$, we can assume that $\max T_{r}^{1} \leq m_{2}$ and $\max T_{s}^{2}=m_{2}+1$. As in the proof of Theorem 2$]$ we put $w=u_{2}-u_{1}$ and find sequences $I_{1}^{1} \leq I_{2}^{1} \leq \cdots \leq I_{n}^{1}$ and 
$I_{1}^{2} \leq I_{2}^{2} \leq \cdots \leq I_{m}^{2}$ of intervals such that $m_{2} \leq \min I_{1}^{j}, j=1,2$,

$$
(1-\gamma)|w|_{+}^{p} \leq \sum_{k=1}^{n}\left(\delta_{+}\left(I_{k}^{1}, w\right)\right)^{p}, \quad(1-\gamma)|w|_{-}^{p} \leq \sum_{k=1}^{m}\left(\delta_{-}\left(I_{k}^{2}, w\right)\right)^{p} .
$$

We can assume that there are $s_{1}, s_{2}$ for which $k_{j}=\min I_{s_{j}}^{j} \leq m_{3}<\max I_{s_{j}}^{j}=l_{j}$, $j=1,2$. Let us put $K_{1}=\left[k_{1}, m_{3}+1\right], K_{2}=\left[m_{3}+1, l_{2}\right]$. We have

$$
\begin{aligned}
\sum_{k<s_{1}}\left(\delta_{+}\left(I_{k}^{1}, w\right)\right)^{p} & +\left(\delta_{+}\left(K_{1}, w\right)\right)^{p}+\sum_{k<s_{2}}\left(\delta_{-}\left(I_{k}^{2}, w\right)\right)^{p} \\
& =\sum_{k<s_{1}}\left(\delta_{-}\left(I_{k}^{1}, u_{1}\right)\right)^{p}+\left(\delta_{+}\left(K_{1}, w\right)\right)^{p}+\sum_{k<s_{2}}\left(\delta_{+}\left(I_{k}^{2}, u_{1}\right)\right)^{p} \\
& \leq\left\|u_{1}\right\|_{ \pm}^{p}<1+\gamma .
\end{aligned}
$$

Similarly

$$
\sum_{k>s_{1}}\left(\delta_{+}\left(I_{k}^{1}, w\right)\right)^{p}+\left(\delta_{-}\left(K_{2}, w\right)\right)^{p}+\sum_{k>s_{2}}\left(\delta_{-}\left(I_{k}^{2}, w\right)\right)^{p}<1+\gamma
$$

Hence

$$
\begin{aligned}
(1-\gamma)\|w\|_{ \pm}^{p} \leq & 2(1+\gamma)+\left(\delta_{+}\left(I_{s_{1}}^{1}, w\right)\right)^{p}-\left(\delta_{+}\left(K_{1}, w\right)\right)^{p} \\
& +\left(\delta_{-}\left(I_{s_{2}}^{2}, w\right)\right)^{p}-\left(\delta_{-}\left(K_{2}, w\right)\right)^{p}
\end{aligned}
$$

But

$$
\|w\|_{ \pm}^{p} \geq\left(\epsilon-\frac{\gamma}{p 2^{p-1}}\right)^{p}>\epsilon^{p}-\gamma
$$

It follows that

$$
\frac{1}{2}\left(\epsilon^{p}-2\right)<\left(\delta_{+}\left(I_{s_{1}}^{1}, w\right)\right)^{p}-\left(\delta_{+}\left(K_{1}, w\right)\right)^{p}+\left(\delta_{-}\left(I_{s_{2}}^{2}, w\right)\right)^{p}-\left(\delta_{-}\left(K_{2}, w\right)\right)^{p} .
$$

We have two cases.

I. $\frac{1}{4}\left(\epsilon^{p}-2\right)<\left(\delta_{+}\left(I_{s_{1}}^{1}, w\right)\right)^{p}-\left(\delta_{+}\left(K_{1}, w\right)\right)^{p}$.

By the mean value theorem

$$
\begin{aligned}
\left(\delta_{+}\left(I_{s_{1}}^{1}, w\right)\right)^{p}-\left(\delta_{+}\left(K_{1}, w\right)\right)^{p} & \leq p\|w\|_{ \pm}^{p-1}\left(\delta_{+}\left(I_{s_{1}}^{1}, w\right)-\delta_{+}\left(K_{1}, w\right)\right) \\
& \leq p 4^{p-1} \frac{1}{2}\left(\left|w\left(l_{1}\right)-\alpha\right|+w\left(l_{1}\right)-\alpha\right) \\
& =p 4^{p-1}\left(u_{2}\left(l_{1}\right)-\alpha\right) .
\end{aligned}
$$

Therefore $8^{-p}\left(\epsilon^{p}-2\right)<u_{2}\left(l_{1}\right)-\alpha$, so we obtain

$$
\begin{aligned}
\left\|u_{2}\right\|_{ \pm}^{p} & \geq \sum_{k=1}^{r}\left(\delta_{+}\left(T_{k}^{1}, u\right)\right)^{p}+\left(u_{2}\left(l_{1}\right)-\alpha\right)^{p}+\sum_{k=1}^{s}\left(\delta_{-}\left(T_{k}^{2}, u\right)\right)^{p} \\
& >(1-\gamma)\|u\|_{ \pm}^{p}+\left(8^{-p}\left(\epsilon^{p}-2\right)\right)^{p} .
\end{aligned}
$$

Consequently

$$
\|u\|_{ \pm}^{p}<(1-\gamma)^{-1}\left(1+\gamma-\left(8^{-p}\left(\epsilon^{p}-2\right)\right)^{p}\right)
$$

which gives (2). 
II. $\frac{1}{4}\left(\epsilon^{p}-2\right)<\left(\delta_{-}\left(I_{s_{2}}^{2}, w\right)\right)^{p}-\left(\delta_{-}\left(K_{2}, w\right)\right)^{p}$.

Similarly as in the previous case we see that

$$
\begin{aligned}
& \left(\delta_{-}\left(I_{s_{2}}^{2}, w\right)\right)^{p}-\left(\delta_{-}\left(K_{2}, w\right)\right)^{p} \\
& \quad \leq p\|w\|_{ \pm}^{p-1}\left(\delta_{-}\left(I_{s_{2}}^{2}, w\right)-\delta_{-}\left(K_{2}, w\right)\right) \\
& \quad \leq p 4^{p-1}\left(-u_{1}\left(k_{2}\right)\right) .
\end{aligned}
$$

Hence $8^{-p}\left(\epsilon^{p}-2\right)<-u_{1}\left(k_{2}\right)$. But

$$
\sum_{k=1}^{r}\left(\delta_{+}\left(T_{k}^{1}, u\right)\right)^{p}+\sum_{k=1}^{s}\left(\delta_{-}\left(T_{k}^{2}, u\right)\right)^{p}+\left(-u_{1}\left(k_{2}\right)\right)^{p} \leq\left\|u_{1}\right\|_{ \pm}^{p} .
$$

Therefore

$$
(1-\gamma)\|u\|_{ \pm}^{p}+\left(8^{-p}\left(\epsilon^{p}-2\right)\right)^{p}<1+\gamma
$$

which implies (2).

From (1) and (2) we see that

$$
\inf \left\{\|x\|: x \in \operatorname{co}\left\{x_{n}\right\}\right\}<\left(1-\frac{1}{3}\left(8^{-p}\left(\epsilon^{p}-2\right)\right)^{p}\right)^{1 / p}+2 \gamma .
$$

Since $\gamma>0$ can be arbitrarily small, we obtain

$$
\Delta(\epsilon) \geq 1-\left(1-\frac{1}{3}\left(8^{-p}\left(\epsilon^{p}-2\right)\right)^{p}\right)^{1 / p}
$$

where $\Delta$ is the modulus of noncompact convexity of $Y_{p}$. This shows that $S_{0}\left(Y_{p}\right) \leq$ $2^{1 / p}$.

\section{REFERENCES}

[1] J. M. Ayerbe Toledano, T. Domínguez Benavides and G. López Acedo, Measures of noncompactness in metric fixed point theory, Birkhäuser Verlag, Basel - Boston - Berlin, 1997. MR 99e: 47070

[2] M. Baronti, E. Casini and P. L. Papini, On average distances and the geometry of Banach spaces, (preprint).

[3] A. Brunel and L. Sucheston, On B-convex Banach spaces, Math. System Theory 7 (1974), 294-299. MR 55:11004

[4] J. Burlak, R. Rankin and A. Robertson, The packing of spheres in the space $l_{p}$, Proc. Glasgow Math. Assoc. 4 (1958), 22-25. MR 22:9917

[5] M. M. Day, Uniform convexity in factor and conjugate spaces, Ann. of Math., 45 (1944), 375-385. MR 6:69c

[6] T. Domínguez Benavides and G. López Acedo, Lower bounds for normal structure coefficients, Proc. Roy. Soc. Edinburgh Sect. A, 121 (1992), 245-252. MR 93i:46025

[7] J. Elton and E. Odell, The unit ball of every infinite dimensional normed linear space contains $a(1+\epsilon)$-separated sequence, Colloq. Math. 44 (1981), 105-109. MR 82k:46025

[8] K. Goebel and W. A. Kirk, Topics in metric fixed point theory, Cambridge University Press, Cambridge, 1990. MR 92c:47070

[9] R. Huff, Banach spaces which are nearly uniformly convex, Rocky Mountain J. Math., 10 (1980), 743-749. MR 82b:46016

[10] R. C. James, Bases and reflexivity of Banach spaces, Ann. of Math., 52 (1950), 518-527. MR 12:616b

[11] R. C. James, A non-reflexive Banach space isometric with its second conjugate space, Proc. Nat. Sci. U. S. A., 37 (1951), 174-177. MR 13:356d

[12] R. C. James, Weak compactness and reflexivity, Israel J. Math., 2 (1964), 101-119. MR 31:585

[13] R. C. James, Uniformly non-square Banach spaces, Ann. of Math., 80 (1964), 542-550. MR 30:4139 
[14] C. A. Kottman, Packing and reflexivity in Banach spaces, Trans. Amer. Math. Soc., 150 (1970), 565-576. MR 42:827]

[15] J. H. Wells and L. R. Williams, Embeddings and extensions in analysis, Springer-Verlag, Berlin, 1975. MR 57:1092

Department of Mathematics, M. Curie-SkŁodowska University, 20-031 Lublin, Poland

E-mail address: akryczka@golem.umcs.lublin.pl

Department of Mathematics, M. Curie-Skeodowska University, 20-031 Lublin, Poland

E-mail address: bsprus@golem.umcs.lublin.pl 